\title{
Mean-field studies of time reversal breaking states in super-heavy nuclei with the Gogny force
}

\author{
L.M. Robledo ${ }^{1, a)}$ \\ ${ }^{1}$ Departamento Física Teórica, Facultad de Ciencias, Universidad Autónoma de Madrid, E-28049 Madrid, Spain \\ a)Corresponding author: luis.robledo@uam.es \\ URL: http://gamma.ft.uam.es/robledo
}

\begin{abstract}
Recent progress on the description of time reversal breaking (odd mass and multi-quasiparticle excitation) states in super-heavy nuclei within a mean field framework and using several flavors of the Gogny interaction is reported. The study includes ground and excited states in selected odd mass isotopes of nobelium and mendelevium as well as high $K$ isomeric states in ${ }^{254} \mathrm{No}$. These are two and four-quasiparticle excitations that are treated in the same self-consistent HFB plus blocking framework as the odd mass states.
\end{abstract}

\section{INTRODUCTION}

For those nuclei with $Z$ values in excess of 92 the Coulomb repulsion wins the competition with the nuclear interaction (in the form of surface tension) and they become unstable against spontaneous fission. This pure semi-classical argument based on energetic considerations concerning the initial (usually the ground state) and final (two fragment) configurations is not enough to understand the dynamics of fission. It has to be supplemented with subtle quantum mechanics effects associated to the shell structure of the underlying nuclear mean field that are very important in the dynamical evolution of the nucleus between the initial and final states. These quantum mechanics effects are responsible for the wealth of long lived nuclei with $Z>92$ and play an essential role in the stability of super-heavy nuclei. The experimental understanding of those effects is based mostly on the analysis of one quasi-particle excitations typical of odd mass nuclei: the spectrum of those systems reveals the distribution of single particle levels around the Fermi level. Recently, experimental studies of the nucleus ${ }^{254}$ No have revealed $[1,2,3]$ how the distribution and properties of high $K$ isomers in even-even nuclei can also help to understand the single particle level distribution.

\section{THEORETICAL FRAMEWORK}

The present theoretical framework is based on the well known Hartree- Fock - Bogoliubov (HFB) method commonly used in nuclear physics $[4,5]$. It relies on the canonical Bogoliubov transformation

$$
\left(\begin{array}{c}
\beta \\
\beta^{\dagger}
\end{array}\right)=\left(\begin{array}{cc}
U^{+} & V^{+} \\
V^{T} & U^{T}
\end{array}\right)\left(\begin{array}{c}
c \\
c^{\dagger}
\end{array}\right)
$$

that defines quasiparticle creation and annihilation operators $\beta_{\mu}^{\dagger}$ and $\beta_{\mu}$ in terms of a convenient single particle basis defined by $c_{i}^{\dagger}$ and $c_{i}$. The Bogoliubov amplitudes $U$ and $V$ are used to define the block matrix $W$ defined above. The HFB wave function $|\Psi\rangle$ is defined as the vacuum of the quasiparticle annihilation operators $\beta_{\mu}|\Psi\rangle=0$. For even particle number systems the HFB wave function can be written in BCS form

$$
|\Psi\rangle=\prod_{v}\left(u_{v}+v_{v} a_{v}^{\dagger} a_{v}^{\dagger}\right)
$$


where the occupancies $v_{v}$ and $u_{v}\left(u_{v}^{2}+v_{v}^{2}=\right)$ and canonical basis creation operators $a_{v}^{\dagger}$ are introduced. The connection between the $U$ and $V$ amplitudes and the above quantities is given by the Bloch-Messiah theorem [5]. The above expression shows that in the even particle number case the symmetry breaking wave function is a linear combination of Slater determinants with an even number of particles (even number parity). To describe a system with an odd number of particles a blocked wave function of the form $\left|\Psi_{\mu_{0}}\right\rangle=\beta_{\mu_{0}}|\Psi\rangle$ is required. It is a linear combination of states with an odd number of particles (odd number parity), it is not invariant under time reversal and is the vacuum of the set of operators

$$
\beta_{1}, \ldots, \beta_{\mu_{0}-1}, \beta_{\mu_{0}}^{\dagger}, \beta_{\mu_{0}+1}, \ldots, \beta_{N} .
$$

The new quasiparticle vacuum can be obtained from the old one $[6,4,7,7,8]$ by swapping the column $\mu_{0}$ of the $U$ and $V$ amplitudes, what converts the $\beta_{\mu_{0}}^{\dagger}$ quasiparticle operator into the $\beta_{\mu_{0}}$ one. The swapping can be implemented also as a swapping of columns in the $W$ block matrix defined above. This is an important point as all the quantities entering the HFB equation in one way or another can be related to that $W$ matrix and therefore the swapping takes care of all the particularities of the blocking procedure straightforwardly. Another peculiarity of the blocked HFB method is that the non-linear character of the blocked equation does not guarantee that blocking the quasiparticle with the lowest quasiparticle energy necessarily leads to the lowest-energy self-consistent solution. The energy gains resulting of such rearrangement are of the order of a few hundred $\mathrm{keV}$, an apparently small quantity, but they are relevant in the determination of the spin and parity of the ground state because of the large number of low lying excited states in odd systems. From a practical standpoint, this peculiarity forces to consider many blocked quasiparticles in order to insure that the ground state is reached $[9,10]$. As a consequence, it is very important to have a robust and efficient method for solving odd-A systems for global applications such as the construction of theoretical mass tables [11, 12, 13, 14]. The blocked wave functions break time reversal invariance and therefore their associated densities $\rho$ and $\kappa$ also break this symmetry. As a consequence, the induced Hartree- Fock and pairing fields also break time reversal invariance. The breaking of time reversal has as a consequence that several terms in the HF and pairing fields that are by zero by construction in even systems have to be considered. In order to consider those extra terms and therefore handle time reversal breaking wave functions, a new computer code named "atb" has been developed [15] as a generalization of an existing axially symmetric solver (HFBax) for the Gogny force. The "atb" code is general enough as to be able to also consider multi-quasiparticle excitations built on top of an even or odd mass ground state. The main advantage of preserving axial symmetry is the $K$ quantum number, that enormously simplifies some intrinsic difficulties associated to the loss of orthogonality. It also allows to easily assign an angular momentum quantum number to the different states obtained by identifying it with the corresponding $K$ value. Parity is allowed to break but in most of the cases is practically a good symmetry of the system and therefore it can be used as an additional quantum number characterizing the different quantum states.
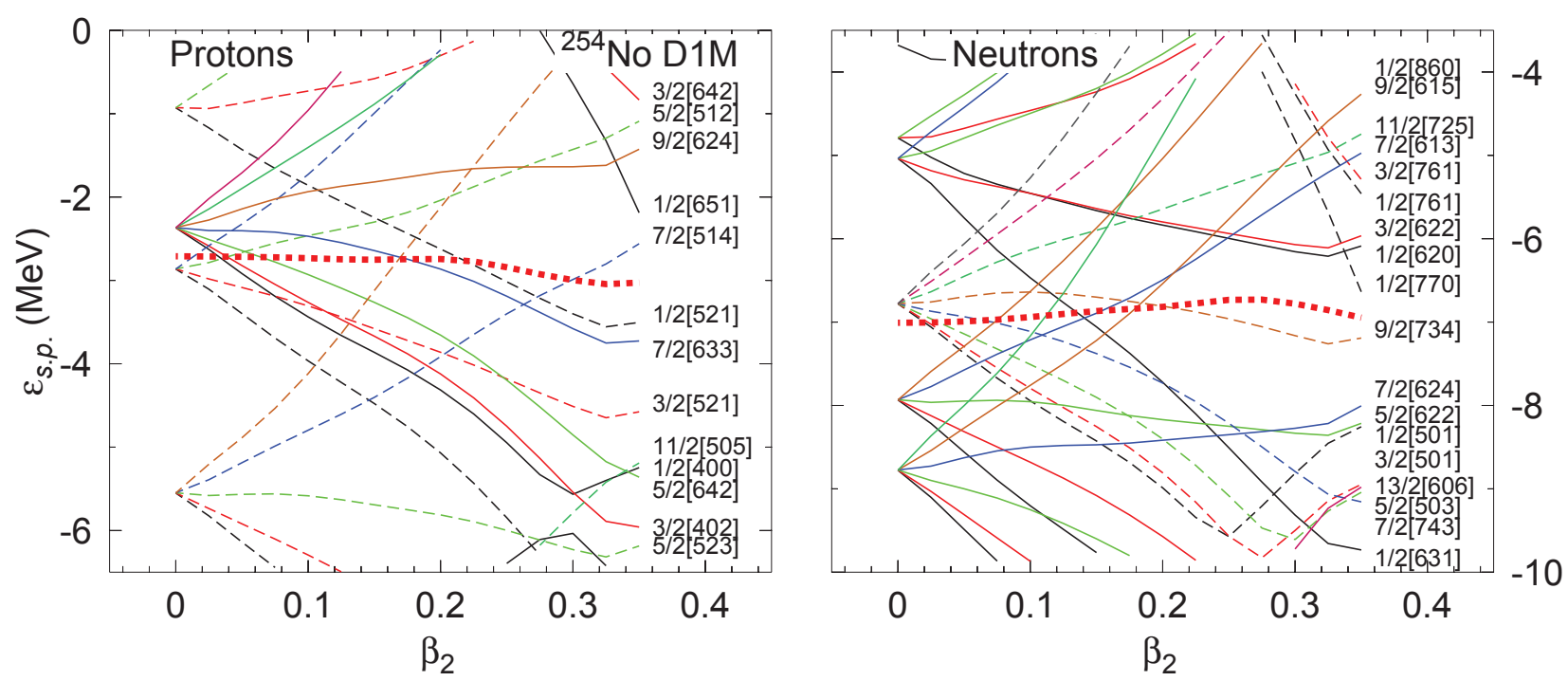

FIGURE 1. Proton and neutron single particle energies are plotted as a function of the deformation parameter $\beta_{2}$ for the ${ }^{254}$ No nucleus. The results are obtained with the D1M parametrization of the Gogny force 
The computer code "atb" uses a generalization of the gradient method to consider blocked HFB wave functions [8] and is able to handle not only constraints on mean values of one or two body operators but also constraints on orthogonality to given wave functions. The latter is a very crucial constraint as the solution of the HFB equation for blocked quasiparticles with the same $K$ quantum number often converge to the same final state (usually with the lowest energy compatible with that $K$ value).

The interaction used is the finite range Gogny pseudo-potential with the D1M [16] and D1S [17] parametrizations. The finite range of the force allows to treat pairing correlations in a consistent way. This is an important asset in the present calculation because its outcome strongly depend on pairing. Both parametrizations have been used recently to describe odd mass systems in the equal filling approximation with great success [9, 10]. For a recent study in the super-heavy region using full blocking refer to Ref [18].

An approximation commonly used to avoid the nuisances of full blocking is the so called "perturbative quasiparticle approximation" where an HFB calculation is carried out to obtain a wave function with even number parity but constrained to have the correct number of protons and neutrons (one of them an odd number). Let us denote by $E_{\text {ref }}$ the energy of such state. The energy of the one quasiparticle states corresponding to the excitation spectra in the odd mass system is taken as the sum of the reference energy $E_{\text {ref }}$ plus the corresponding one quasiparticle energies obtained by diagonalizing the matrix $H^{11}-\lambda N^{11}$ (see [5] for notation).

\section{RESULTS}

A few results involving super-heavy nuclei will be used to illustrate the possibilities of the HFB method with blocking when used with the Gogny force. First we will discuss the relevant single particle levels obtained in ${ }^{254}$ No with the D1M parametrization. Next, results for the odd-A isotopes ${ }_{101}^{251-253-255} \mathrm{Md}$ and ${ }_{102}^{253-255-257}$ No will be discussed and compared to experimental data. Finally, two-quasiparticle and four-quasiparticle high $K$ isomers in ${ }^{254}$ No are discussed and their excitation energies compared to experimental data.

\section{Single particle energies in ${ }^{254} \mathrm{No}$}

Single particle energies (spe) as a function of the $\beta_{2}$ quadrupole deformation parameter are obtained by diagonalizing the Hartree-Fock hamiltonian $h$ with the corresponding Lagrange term subtracted $\lambda \hat{Q}_{20}$. The eigenvector of $h$ can be labeled with the asymptotic Nilsson quantum number of the HO orbital with the largest occupancy. In Fig 1 the spe's corresponding to the nucleus ${ }^{254}$ No and obtained with the D1M parametrization are depicted for both protons and neutrons. In the proton side, we observe near the Fermi level and at the ground state deformation $\left(\beta_{2}=0.28\right)$ the negative parity $7 / 2[514], 1 / 2[521]$ and positive parity $7 / 2[633]$ orbitals responsible for the lowest lying spectra of the $Z=101 \mathrm{Md}$ isotopes discussed below. In the neutron side, the 9/2[734] orbital is below the Fermi level at $\beta_{2}=0.28$, above it we find the $1 / 2[770], 2[620]$ and 3/2[622] orbitals responsible for the spectra of the odd-A nobelium isotopes discussed below. A deformed proton gap corresponding to $Z=104$ is observed at $\beta_{2}=0.3$ whereas two deformed gaps at $N=150$ and $N=152$ can also be noticed.

$$
\text { Odd-A }{ }_{101}^{251-253-255} \text { Md and }{ }_{102}^{253-255-257} \text { No isotopes }
$$

Full blocking calculations as described in the previous section have been carried out for the $A=253-257$ isotopes of No $(Z=102)$ and the $A=251-255$ isotopes of $M d(Z=101)$. In the upper and middle panels of Fig 2 the No results obtained with the D1S (upper panels) and D1M (middle panels) parametrizations of the Gogny force are shown. For each nucleus, two sets of calculations (left columns) are compared among them and with experimental data. In the leftmost columns the results of the "perturbative quasiparticle calculation" (see previous section) are given. In the middle column the excitation energies of the full blocking calculations are depicted whereas in the rightmost columns the experimental data is presented. There are two noticeable facts concerning the "perturbative" calculation: first, the ground state energy is higher (less bound) than in the full blocking case (with D1S the values are $255 \mathrm{keV}, 161 \mathrm{keV}$ and $152 \mathrm{keV}$ for ${ }^{253} \mathrm{No},{ }^{255} \mathrm{No}$ and ${ }^{257} \mathrm{No}$, respectively). This underbinding of the perturbative results as compared to full blocking implies a larger pairing gap (by the same amount) in the former. This is consistent with the greater efficiency of the full blocking procedure to quench pairing correlations. The second noticeable fact is that, although the ordering in the sequence of "perturbative" levels coincides with the one of full blocking, the excitation energies can differ up to $200-300 \mathrm{keV}$ which is a large amount. Therefore, the "perturbative" approximation can not be considered as a reliable alternative to full blocking if a precision smaller than $200-300 \mathrm{keV}$ is required. Concerning the predictions 

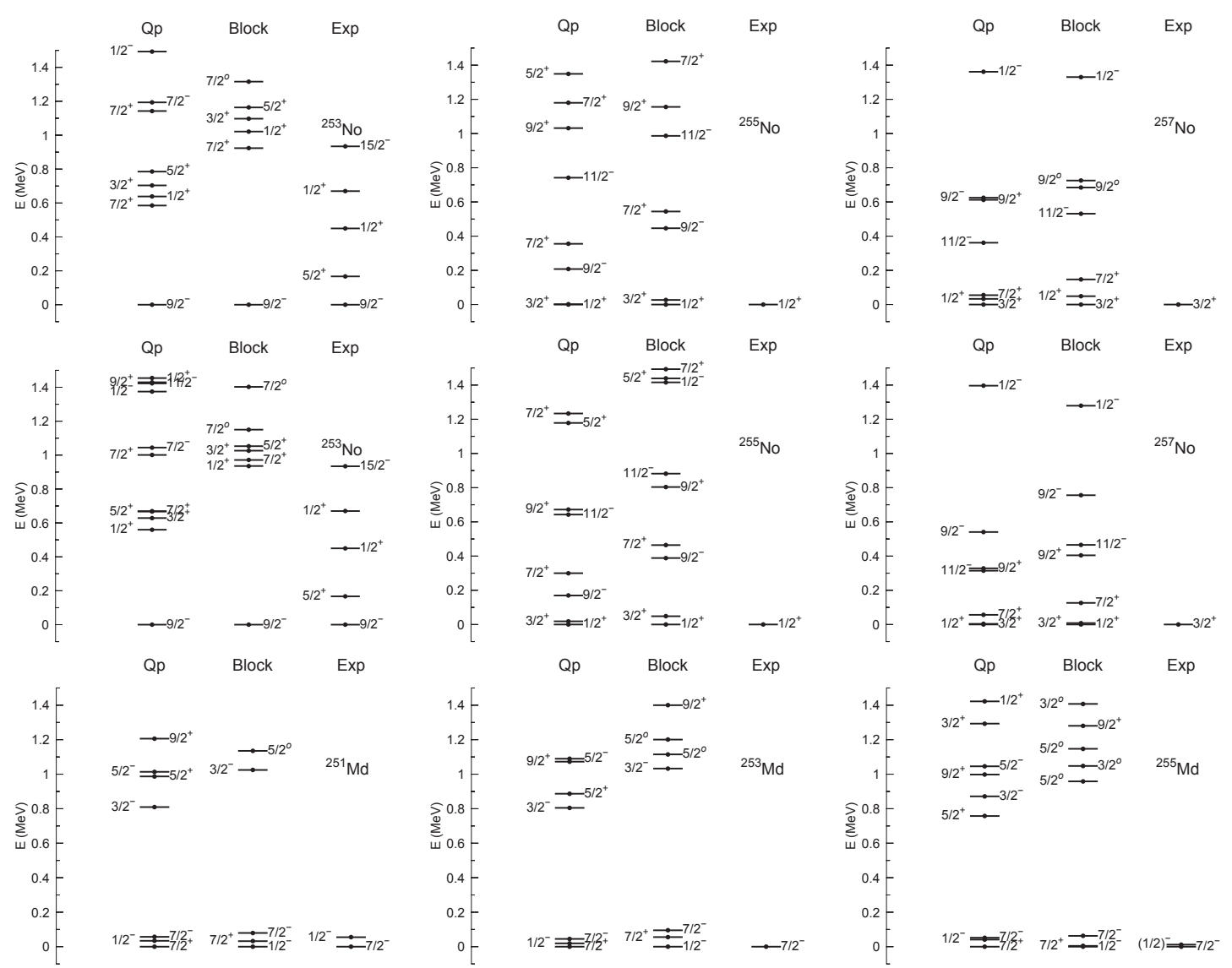

FIGURE 2. Theoretical and experimental spectra of the considered isotopes. Upper (middle) panels show results for the No isotopes obtained with the D1S (D1M) parametrizations of Gogny. In the lower panels, results for the Md isotopes computed with the Gogny D1S force are shown. For each nucleus, the column marked as "Qp" is for the spectrum obtained in the "perturbative quasiparticle approximation" and the one marked with "Block" is for the full blocking spectrum. Levels with an "o" for the parity quantum number have a slight octupole deformation that prevents a clear parity assignment. Experimental data taken from [19].

of the two parametrizations, the predicted ordering of levels is almost the same (see for instance the $9 / 2^{+}$in ${ }^{257} \mathrm{No}$ ) in the two cases but there are typically quantitative differences of the order of $100-200 \mathrm{keV}$ between them. This is an indication of the variability of results with the interaction used. Finally, the quenching of pairing correlations induced by blocking is severe but depends on the specific level. Proton pairing correlations are not quenched for the odd No as expected. For neutrons, taking the ${ }^{255}$ No as an example, the quenching in the ground state $1 / 2^{+}$is very efficient and neutron pairing correlations are almost gone with a negligible $\left\langle\Delta N^{2}\right\rangle /\left\langle\Delta N^{2}\right\rangle_{\text {ref }}$ ratio $\left(\left\langle\Delta N^{2}\right\rangle_{\text {ref }}\right.$ is the neutron number fluctuation of the even number parity wave function with the $\mathrm{Z}$ and $\mathrm{N}$ values of ${ }^{255} \mathrm{No}$ ). The same happens for the first excited state $3 / 2^{+}$. However pairing correlations remain almost unquenched for the $9 / 2^{-}$state with a $\left\langle\Delta N^{2}\right\rangle /\left\langle\Delta N^{2}\right\rangle_{\text {ref }}$ ratio of 0.95 . For the $7 / 2^{+}$the ratio is 0.54 and takes the value 0.41 for the $11 / 2^{-}$. These typical values show a large variability that points to the importance of a good description of beyond mean field pairing correlations for a proper description of the states.

In the comparison with experimental data we observe that the spin and parity of the ground state is well reproduced in the three isotopes but the experimental excitation spectrum in ${ }^{253}$ No is much lower in energy than the theoretical predictions. More experimental data in ${ }^{255} \mathrm{No}$ and ${ }^{257} \mathrm{No}$ is required to asses the quality of the calculations in reproducing the experimental spectra.

In the Md case, depicted in the lower panel of Fig 2, protons are the type of nucleon to be blocked and therefore the spectrum is not expected to change much with neutron number (i.e. among different isotopes). This is observed in the figure, where we notice a close resemblance of the spectra of the three isotopes considered. Concerning the 
comparison between the ground state energy obtained with the "perturbative" approximation and the one of full blocking, $199 \mathrm{keV}, 160 \mathrm{keV}, 185 \mathrm{keV}$ are gained in binding energy (with the corresponding reduction of the pairing gap) when full blocking is considered. The ground state and lowest excited states of the three nuclei are mostly determined by the the negative parity $7 / 2[514], 1 / 2[521]$ and positive parity $7 / 2[633]$ orbitals near the Fermi level. Given the almost degeneracy of the three orbitals observed in the calculations, the predictions for the spin and parity of the ground state do not match in all the cases the experimental observations.

\section{High $K$ isomers in ${ }^{254}$ No}

Given the relatively good description of the ground state and low lying band heads in the odd mass nuclei discussed above one may wonder how good the agreement would be in other situations using the same building blocks. To answer this question we are going to use the properties of the high $K$ isomers recently found in ${ }^{254}$ No [1, 2, 3]. A two proton quasiparticle excitation $K^{\pi}=3^{-}$, two neutron ones $K^{\pi}=8^{-}$and $10^{+}$as well as a two-proton two-neutron high $K$ isomeric $16^{+}$state have been experimentally characterized. The excitation energy of those states is given in Table 1 along with our predictions using D1M and D1S. A good agreement with experiment is obtained for the $3^{-}$ and $10^{+}$states, and a reasonable one for the $8^{-}$and $16^{+}$states with an overestimation of $25 \%$. The Nilsson orbitals involved in each of the excited states are the $\pi 7 / 2[514] 1 / 2[521]$ for the $3^{-}$, the $v 9 / 2[734] 7 / 2[624]$ for the $8^{-}$, the $\nu 9 / 2[734] 11 / 2[725]$ for the $10^{+}$and finally the $\pi 9 / 2[624] 7 / 2[514] \nu 9 / 2[734] 7 / 2[624]$ for the $16^{+}$.

TABLE 1. Excitation energy (in MeV) of several two and four quasiparticle $K$ isomers in ${ }^{254}$ No. Experimental data taken from [3].

\begin{tabular}{lcccc}
\hline & $3^{-}$ & $8^{+}$ & $10^{-}$ & $16^{+}$ \\
Experiment & 0.988 & 1.296 & 2.012 & 2.928 \\
Gogny D1S & 1.162 & 1.805 & 2.287 & 4.040 \\
Gogny D1M & 1.194 & 1.768 & 2.212 & 3.950 \\
\hline
\end{tabular}

Theoretical models based on macroscopic-microscopic methods with the Wood-Saxon potential provide a good agreement with experiment in this case with differences in excitation energies never exceeding $200 \mathrm{keV}$ (see Refs $[2,3]$ for an overview and comparison with experimental data). The projected shell model [1] also provides with a good description of experimental data but it has to be kept in mind that the relevant Nilsson orbital energies are adjusted in each case. On the other hand, similar calculations to the ones presented here but using the SLy4 Skyrme interaction and density dependent pairing are unable to reproduce the features of high $K$ isomers shown in ${ }^{254}$ No [20].

\section{CONCLUSIONS}

In this paper we have analyzed the ability of the HFB method with blocking to reproduce the low lying spectrum of several nobelium and mendelevium odd mass isotopes around ${ }^{254}$ No. The well known D1S and D1M parametrizations of the Gogny force have been used in the calculations. As a consequence of the reasonable agreement obtained with the scarce experimental data, a study of high $K$ two and four quasiparticle excitations in ${ }^{254}$ No was carried out. Again, a reasonable agreement with the experimental excitation energies of those states is observed. The worse case corresponds to the $K=16^{+}$isomer where the predictions for the excitation energy are around $25 \%$ larger than the experimental value. For the other states the agreement is slightly better. The limitation to axially symmetric shapes restricts the study to the band heads of rotational bands. The reason is the cranking $\omega J_{x}$ term required to build up the collective angular momentum of rotational bands in a HFB scheme that mixes the $K$ quantum number. Without those rotational states, it is not possible to analyze the decay out of the high $K$ isomers.

It is comforting to observe the reasonable agreement with experiment, given the universal character of the forces considered: they are used with reasonable success to describe a wealth of physical situations all over the nuclide chart. To improve the performance of the forces without local adjustment of parameters, new, more flexible interactions are required, incorporating, perhaps, a richer and finite-range spin-orbit interaction and a flexible tensor potential. However, one should not forget that this is a mean field study and therefore correlations beyond the mean field could alter substantially the whole picture obtained here. In this respect, one cannot forget the recent study of [21] where it was shown that dynamical octupole correlations are present in all even-even nuclei in the nuclide chart. Polarization effects in odd mass states and multi-quasiparticle excitations lead to an enhanced octupole softness and therefore 
to a more important role of dynamical correlations in the description of those states. Additionally, one should not forget that the blocked states tend to quench pairing correlations into a regime where beyond mean field correlations associated to symmetry restoration can also play a relevant role.

\section{ACKNOWLEDGMENTS}

This work has been supported in part by the MINECO (Spain) grants Nos. FPA2012-34694 and FIS2012-34479, and by the Consolider-Ingenio 2010 program MULTIDARK CSD2009-00064.

\section{REFERENCES}

[1] R.-D. Herzberg, P. T. Greenlees, P. A. Butler, G. D. Jones, M. Venhart, I. G. Darby, S. Eeckhaudt, K. Eskola, T. Grahn, C. Gray-Jones, F. P. Hessberger, P. Jones, R. Julin, S. Juutinen, S. Ketelhut, W. Korten, M. Leino, A.-P. Leppanen, S. Moon, M. Nyman, R. D. Page, J. Pakarinen, A. Pritchard, P. Rahkila, J. Saren, C. Scholey, A. Steer, Y. Sun, C. Theisen, and J. Uusitalo, "bibfield journal "bibinfo journal Nature" "textbf "bibinfo volume 442," "unskip" "bibinfo pages 896-899 ("bibinfo year 2006).

[2] S. K. Tandel, T. L. Khoo, D. Seweryniak, G. Mukherjee, I. Ahmad, B. Back, R. Blinstrup, M. P. Carpenter, J. Chapman, P. Chowdhury, C. N. Davids, A. A. Hecht, A. Heinz, P. Ikin, R. V. F. Janssens, F. G. Kondev, T. Lauritsen, C. J. Lister, E. F. Moore, D. Peterson, P. Reiter, U. S. Tandel, X. Wang, and S. Zhu, "bibfield journal "bibinfo journal Phys. Rev. Lett.“ "textbf "bibinfo volume 97,“" p." "bibinfo pages 082502 ("bibinfo year 2006).

[3] R. Clark, K. Gregorich, J. Berryman, M. Ali, J. Allmond, C. Beausang, M. Cromaz, M. Deleplanque, I. Dragojevi, J. Dvorak, P. Ellison, P. Fallon, M. Garcia, J. Gates, S. Gros, H. Jeppesen, D. Kaji, I. Lee, A. Macchiavelli, K. Morimoto, H. Nitsche, S. Paschalis, M. Petri, L. Stavsetra, F. Stephens, H. Watanabe, and M. Wiedeking, "bibfield journal "bibinfo journal Physics Letters B" "textbf "bibinfo volume 690," "unskip" "bibinfo pages $19-24$ ("bibinfo year 2010).

[4] H. Mang, "bibfield journal "bibinfo journal Physics Reports" "textbf "bibinfo volume 18," "unskip" "bibinfo pages 325 - 368 ("bibinfo year 1975).

[5] P. Ring and P. Schuck, The nuclear many body problem (Springer, Berlin, 1980).

[6] B. Banerjee, H. Mang, and P. Ring, "bibfield journal "bibinfo journal Nuclear Physics A" "textbf "bibinfo volume 215," "unskip" "bibinfo pages 366 - 382 ("bibinfo year 1973).

[7] G. Bertsch, J. Dobaczewski, W. Nazarewicz, and J. Pei, "bibfield journal "bibinfo journal Phys. Rev. A" "textbf "bibinfo volume 79," p." "bibinfo pages 043602 ("bibinfo year 2009).

[8] L. M. Robledo and G. F. Bertsch, "Pairing in finite systems: beyond the hfb theory," in Fifty Years of Nuclear BCS: Pairing in Finite Systems, edited by R. A. Broglia and V. Zelevinsky (World Scientific, Singapore, 2013).

[9] R. Rodriguez-Guzman, P. Sarriguren, and L. M. Robledo, "bibfield journal "bibinfo journal Phys. Rev. C" "textbf "bibinfo volume 82," p." "bibinfo pages 044318 ("bibinfo year 2010"natexlaba).

[10] R. Rodriguez-Guzman, P. Sarriguren, and L. M. Robledo, "bibfield journal "bibinfo journal Phys. Rev. C" "textbf "bibinfo volume 82," p." "bibinfo pages 061302 ("bibinfo year 2010"natexlabb).

[11] N. Schunck, J. Dobaczewski, J. McDonnell, J. Moré, W. Nazarewicz, J. Sarich, and M. V. Stoitsov, "bibfield journal "bibinfo journal Phys. Rev. C" "textbf "bibinfo volume 81," p." "bibinfo pages 024316 ("bibinfo year 2010).

[12] S. Hilaire and M. Girod, "bibfield journal "bibinfo journal The European Physical Journal A - Hadrons and Nuclei"“ "textbf "bibinfo volume 33," "unskip" "bibinfo pages 237-241 ("bibinfo year 2005).

[13] E. V. Litvinova and A. V. Afanasjev, "bibfield journal "bibinfo journal Phys. Rev. C" "textbf "bibinfo volume 84," p." "bibinfo pages 014305 ("bibinfo year 2011).

[14] A. Afanasjev and S. Shawaqfeh, "bibfield journal "bibinfo journal Physics Letters B" "textbf "bibinfo volume 706," “unskip" "bibinfo pages 177 - 182 ("bibinfo year 2011).

[15] L. M. Robledo, R. Bernard, and G. F. Bertsch, "bibfield journal "bibinfo journal Phys. Rev. C" "textbf "bibinfo volume 86," p." "bibinfo pages 064313 ("bibinfo year 2012).

[16] S. Goriely, S. Hilaire, M. Girod, and S. Péru, Phys. Rev. Lett. 102, p. 242501 (2009).

[17] J. F. Berger, M. Girod, and D. Gogny, Nucl. Phys. A 502, p. 85 (1989). 
[18] J. Dobaczewski, A. Afanasjev, M. Bender, L. Robledo, and Y. Shi, "bibfield journal "bibinfo journal Nuclear Physics A" "unskip "bibinfo pages - ("bibinfo year 2015).

[19] E. Browne and J. Tuli, "bibfield journal "bibinfo journal Nuclear Data Sheets" "textbf "bibinfo volume 114," "unskip" "bibinfo pages 1041 - 1185 ("bibinfo year 2013).

[20] M. Bender, P. Bonche, T. Duguet, and P.-H. Heenen, "bibfield journal "bibinfo journal Nuclear Physics A" "textbf "bibinfo volume 723,“" "unskip"“"bibinfo pages 354 - 364 ("bibinfo year 2003).

[21] L. M. Robledo, "bibfield journal "bibinfo journal Journal of Physics G: Nuclear and Particle Physics"“ "textbf "bibinfo volume 42," p." "bibinfo pages 055109 ("bibinfo year 2015). 